\title{
Subdural Empyema
}

\author{
John Gorman, Nikkie Randhawa, Daniel Mendelsohn, Christopher R. Honey, \\ Manraj K. S. Heran, Silke Appel-Cresswell
}

Keywords: Neuroinfectious disease, Neuroimmunology, Neurosurgery, Surgical pathology

doi:10.1017/cjn.2018.67

Can J Neurol Sci. 2018; 45: 566-567

A 69-year-old woman on long-term immunosuppressive therapy (tacrolimus) for liver transplantation presented to the hospital with headache, nausea and vomiting 2 weeks after a minor closed head injury. Neurological examination was normal but CT imaging was consistent with a 6-mm left frontoparietal subdural collection with minimal mass effect. On the following day, she developed slowed dysarthric speech, word-finding difficulty and paraphasic errors with intact comprehension. She also had a right facial droop and mild pronator drift of the right arm. Her white blood cell count and C-reactive peptide were elevated at $12.2 \times 10^{9} / \mathrm{L}$ and $121 \mathrm{mg} / \mathrm{dl}$, respectively. Investigations for seizure and stroke were negative. On day 3 of her presentation, her language deficit progressed to mutism and she became febrile.

Clinically, this case posed several challenges. The phenomenology suggested localization ipsilateral to the subdural collection, which was unchanged in size on follow-up non-contrast CT head. There was, however, an increase in local mass effect, reflected by increased sulcal effacement adjacent to the collection. Contrast-enhanced imaging was contraindicated owing to acuteon-chronic renal dysfunction from a urinary tract infection (UTI). There was hyperintensity of the fluid collection on diffusionweighted MRI, corresponding to restricted diffusion on the apparent diffusion coefficient map. Susceptibility-weighted MRI demonstrated heterogeneity within the collection, without marked signal drop, as would be typical of a collection dominated by evolving blood products (Figure 1).

Although the patient's language dysfunction was progressive, she did not become febrile until the third day of presentation. A subdural empyema (SDE) was suspected, she was treated with intravenous antibiotics and craniotomy was performed for evacuation of the subdural collection, with frank pus identified (Figure 2). The origin of the SDE was thought to be due to infection of a presumed subdural hematoma (SDH) acquired with the fall and minor head injury 2 weeks earlier, and subsequent infectious spread from a UTI on the background of immunosuppression. Both intraoperative cultures and initial urine cultures were remarkable for heavy growth of Escherichia coli. The patient recovered postoperatively with no recurrence and was discharged to a rehabilitation facility on oral antibiotics to complete an 8-week course.

Subdural empyema is typically caused by a preceding neurosurgical procedure, sinusitis, otitis media, mastoiditis or meningitis. ${ }^{1}$ Secondary infection of a chronic SDH is an uncommon phenomenon. Previous literature has indicated that this condition is more common in older adults with comorbid disease, including immunologic dysfunction. E. coli is commonly the causative organism of infected SDH, as may have occurred in our patient with a concurrent UTI. ${ }^{2}$ Imaging characteristics of SDE may be subtle compared with the clinical severity, requiring a high index of suspicion for diagnosis. Both contrast-enhanced CT and MRI may show peripheral enhancement. Diffusion-weighted imaging can be helpful in the diagnosis of SDE, ${ }^{3,4}$ and in our patient susceptibility-weighted sequences were also useful in differentiating empyema from hematoma. Once identified, SDE is treated with antibiotic therapy and source control by surgical evacuation. Both burr hole washout and craniotomy have been used, with evidence for lower recurrence and mortality rates after craniotomy. ${ }^{2,3,5}$ Although SDE is rare, a high index of suspicion must be maintained for prompt diagnosis and early surgical intervention, especially in patients with a clinical presentation out of proportion with imaging findings.

\section{ACKNOWLEDGMENT}

SA-C is supported by the Marg Meikle Professorship for Parkinson's disease research.

\section{Disclosures}

All authors have nothing to disclose.

From the Department of Medicine, Division of Neurology, University of British Columbia, Vancouver, British Columbia, Canada (JG, NR, SA-C); Department of Neurology, Alpert Medical School of Brown University and Rhode Island Hospital, Providence, Rhode Island, USA (NR); Department of Surgery, Division of Neurosurgery, University of British Columbia, Vancouver, British Columbia, Canada (DM, CRH); Department of Radiology, Division of Neuroradiology, University of British Columbia, Vancouver, British Columbia, Canada (MKSH).

Received September 6, 2017. Final Revisions Submitted April 8, 2018. Date of ACCEPTANCE APRIL 10, 2018.

Correspondence to: S. Appel-Cresswell, Department of Medicine, Division of Neurology, University of British Columbia, 2221 Wesbrook Mall, Vancouver, BC V6T 2B5, Canada. Email: silkec@ mail.ubc.ca 

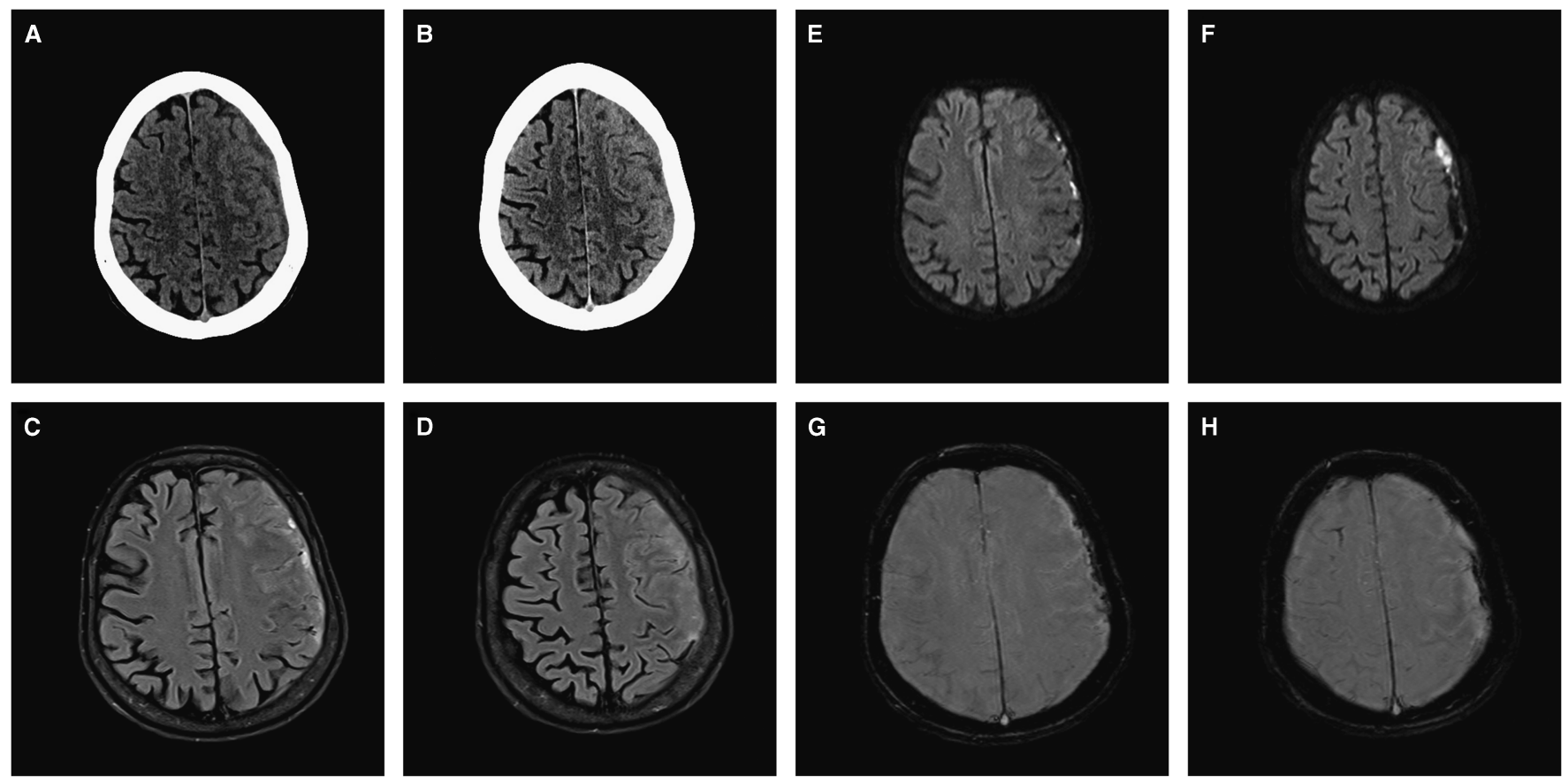

Figure 1: Representative axial images of the subdural collection on non-contrast CT: (A) day 1 of presentation, (B) on day 2 of presentation, FLAIR (C,D), diffusion-weighted MRI $(E, F)$ and susceptibility-weighted MRI $(G, H)$ on day 2 of presentation.
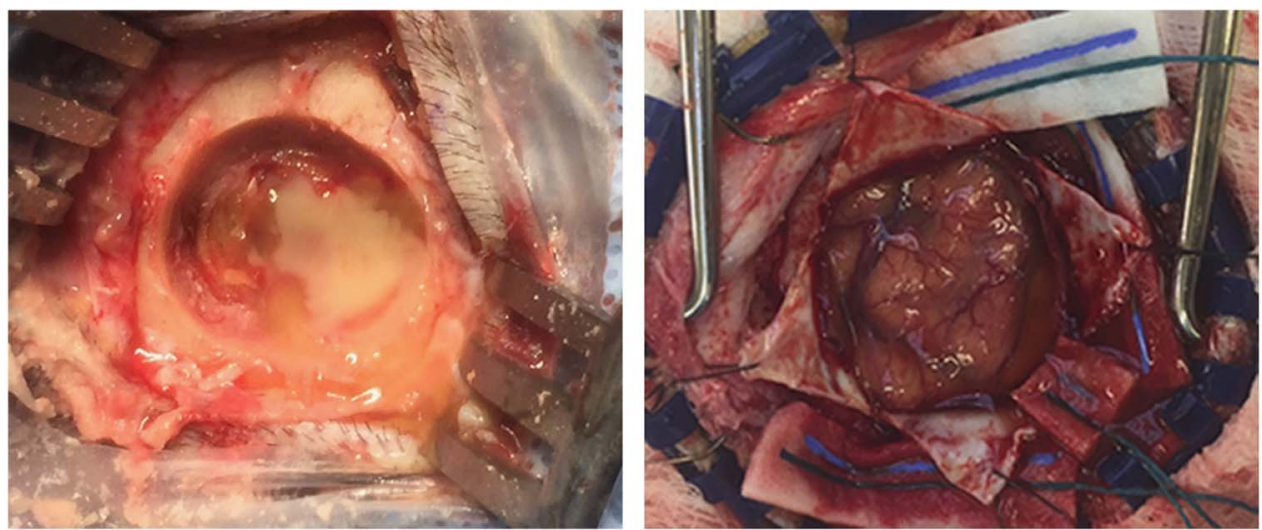

Figure 2: Intraoperative photographs of burr hole (left) converted to craniotomy (right), with frank pus evacuated from the subdural fluid collection.

\section{STatement of Authorship}

JG, NR and SA-C were involved in study concept and design. JG, NR, DM, CRH, MKSH and SA-C contributed to data acquisition. JG drafted the manuscript. JG, NR, DM, CRH, MKSH and SA-C performed critical revision of the manuscript for important intellectual content. SA-C supervised the study.

\section{REFERENCES}

1. French F, Schaefer N, Keijzers G, Barison D, Olson S. Intracranial subdural empyema: a 10-year case series. Ochsner J. 2014;14(2):188-94.
2. Dabdoub CB, Adorno JO, Urbano J, Silveira EN, Orlandi BMM. Review of the management of infected subdural hematoma. World Neurosurg. 2016;87:663.e1-8.

3. Munusamy T, Dinesh SK. Delayed occurrence of Escherichia coli subdural empyema following head injury in an elderly patient: a case report and literature review. J Neurol Surg Rep. 2015;76(1): e79-82.

4. Wong AM, Zimmerman RA, Simon EM, Pollock AN, Bilaniuk LT. Diffusion-weighted MR imaging of subdural empyemas in children. Am J Neuroradiol. 2004;25(6):1016-21.

5. Agrawal A, Timothy J, Pandit L, Shetty L, Shetty JP. A review of subdural empyema and its management. Infect Dis Clin Pract. 2007;15(3):149-53. 